\title{
ARM7 Based Wireless Data Transmission Using Zigbee
}

\author{
Dipali k. Shende ${ }^{1}$, Arun K. Mane ${ }^{2}$, Rahul P. More ${ }^{2}$, Nikhil M. Nawale ${ }^{2}$ \\ Professor, Department of Electronics and Telecommunication, Sinhgad Institute of Technology, Lonavala, \\ Maharashtra, India ${ }^{1}$ \\ Students, Department of Electronics and Telecommunication, Sinhgad Institute of Technology, Lonavala, \\ Maharashtra, India ${ }^{2,3,4}$
}

\begin{abstract}
Voice communication becomes ineffective in the industries \& various places where there is a noisy environment. Industries and other places which offer ineffective voice communication require data transmission in text and image form. The first approach of the project is to draw the pattern on touch screen display which is interfaced with arm7 processor and transmit this pattern using zigbee transmitter. At the receiving side ARM7 processor is interfaced with GLCD (Graphical Liquid Crystal Display) which displays the received pattern. The second approach is to send the bitmap images to the receiver. The bitmap images are stored in the ARM-7 processor's inbuilt EEPROM memory at the transmitter side. The touch screen display along with the GLCD is connected to the ARM7 LPC2148 controller. The ARM processor which is programmed to read the touched values in the real time environment, encode it and sends it to receiver. Whatever the information we want to send is accessed with the help of touch screen and ARM-7 processor and then it is showed on the GLCD at the transmitter. After that we can transmit that image or pattern to the receiver via zigbee transmitter circuit. This project is designed around the ARM-7 processor. The processor fulfils all the requirements related to wireless control and image processing. An inbuilt EEPROM is provided to store the bitmap images.
\end{abstract}

Keywords: Environment, pattern, wireless, zigbee.

\section{INTRODUCTION}

Now days, use of embedded systems in various fields is is sent through the zigbee transmitter circuit. At the increasing rapidly. Each and every day our life is receiver side GLCD and Zigbee receiver circuit is becoming more dependent on 'embedded systems'. This interfaced with the LPC2148 processor. Zigbee receiver includes medical devices, communication field, aerospace, receives the information and then the processor decodes automation industries, railways, e world etc. All of these the information received. GLCD displays the received have wide-ranging impact on society providing privacy, pattern or images. For displaying the pattern the ARM7 security.More than $98 \%$ of processors applied today are in processor decodes the information and then finds out the $\mathrm{X}$ embedded system. The importance of embedded system is and Y co-ordinates of the drawn. The zigbee trans receiver growing continuously. The idea of this project is to circuit used in this project has $100 \mathrm{~m}$ trans receiving range. transmit the drawn text pattern data and images using wireless technology. This can be done by using zigbee Trans receiver.The pattern is drawn on the touch screen display and then it is encoded through arm-7 processor and transmitted by the zigbee transmitter circuit. At the receiving side another zigbee receiver circuit is used for receiving purpose and then received information is decoded by arm7 processor then displays this pattern or text data on GLCD.

\section{SYSTEM DESCRIPTION}

The Fig.1 And Fig.2 Shows the system block diagram. Basically the system consist three component i.e. ARM7 controller (LPC2148), touch screen with GLCD and zigbee module.At the transmitter side, touch screen with the GLCD is interfaced with the LPC2148 processor. Zigbee transmitter circuit is also interfaced with the processor as the RF transmitting device. The pattern is drawn with the help of touch screen on the GLCD. Also the touch screen is very important to fetch images from ARM memory and to send them. The encoded information

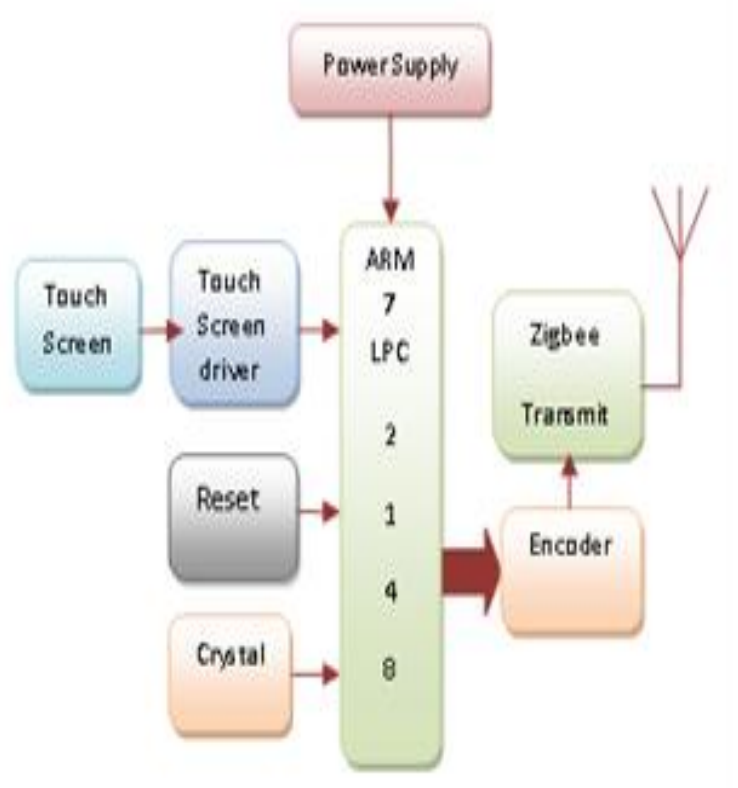

Fig 1. Transmitter block diagram 


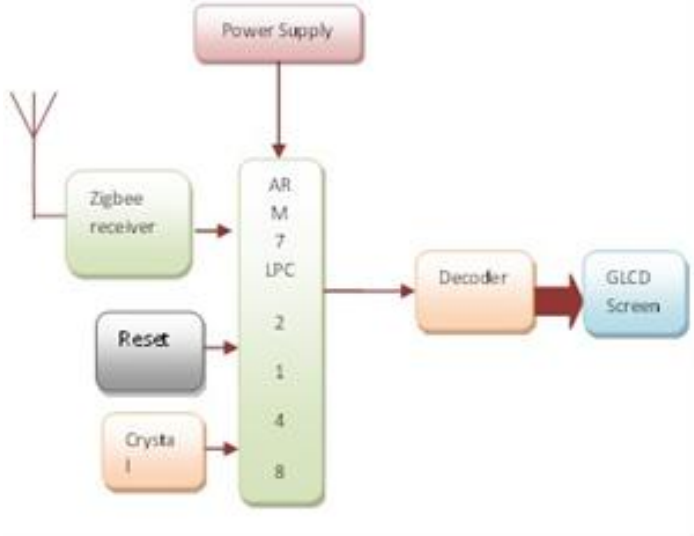

Fig2. Receiver block diagram

\section{A. ARM LPC2148 Processor}

ARM is today's foremost choice available for embedded systems. Over the last few years, the ARM architecture has become worlds one of the most invasive 32-bit architecture in various IC form from various IC manufacturers. ARM7 is one of the micro-controller families which is widely used in embedded system applications. ARM7 processors require significantly fewer transistors than other processors. Hence it reduces power and heating issues also it reduces cost. The ARM-7 processor provides on board serial communication interfaces such as SPI, USB 2.0, SSP to I2C bus, multiple UARTs, on chip SRAM from $8 \mathrm{~KB}$ to $40 \mathrm{~KB}$. These features make this device suitable for voice recognition and low end imaging, soft modems, communication gateways and protocol converters, etc. The ARM-7 processor plays an important role in this system. The touched information encoding at transmitter and decoding at the receiver is done by processor.

\section{B. Touch screen}

A touch screen is an electronic display which is sense the location of a touch within the display region. In this project the 4 wire resistive touch screen display is used. The phrase generally refers to touching the display of the device with a finger and stylus. Fig. 4 shows how to interface the touch panel with the LPC2148 processor.To identify the touched location, wehave to read touch position consecutively i.e. first read $\mathrm{X}$ position and then read the Y position.
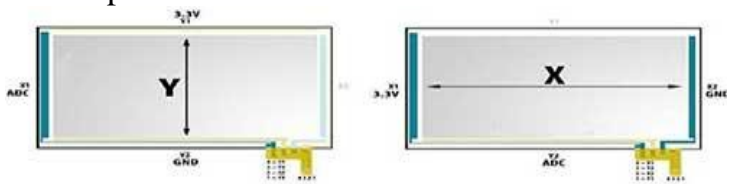

Fig 3. Touch screen foils

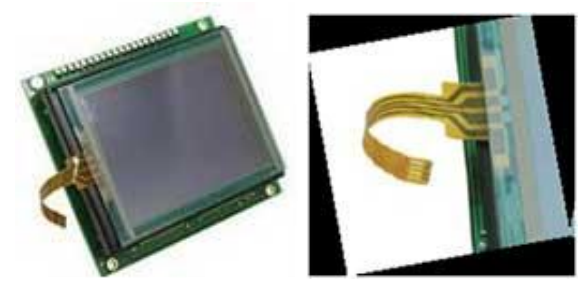

Fig 4. Touchscreen Display
C. Principle of operation

The resistive touch panel is constructed by sandwiching two transparent rigid foils having resistive layer on their inner side. Usually the resistance of the foil should not exceed $1 \mathrm{Kohm}$. Contacts made on the opposite sides of the foils by a flat cable. The process of identifying the touched place point is partitioned into two steps. The first step is to identify the $\mathrm{X}$ co-ordinate and the second one is to identify the $\mathrm{Y}$ co-ordinate. For determining the $\mathrm{X}$ co-ordinate, left contact on the $\mathrm{X}$ surface is connected to ground i.e. 0 voltage levels and the right contact is connected to the power supply. Thus voltage divider is made when we touches on the screen. Y surface bottom contact reads the values of divider. The divider voltage ranges from $0 \mathrm{~V}$ to power supply voltage and it depends upon the $\mathrm{X}$ coordinate. If the touch is nearer to $\mathrm{X}$ surface left contact, then the voltage is taken as $0 \mathrm{~V}$ by the processor. In order to identify $\mathrm{Y}$ co-ordinate of $\mathrm{Y}$ surface then bottom contact of surface $\mathrm{Y}$ is grounded and upper contact is connected to power supply [3].

\section{Graphical Liquid Crystal Display (GLCD)}

In this project the $16 \times 2$ Character LCDs have their own restrictions; they can only display characters of certain magnitudes. Thus the Graphical LCD is used to display modified characters and images. The Graphical LCD is use in various applications; they are used in video games, mobile phones, and lifts etc. as display units. Various graphical LCDs are available in the market with different sizes.

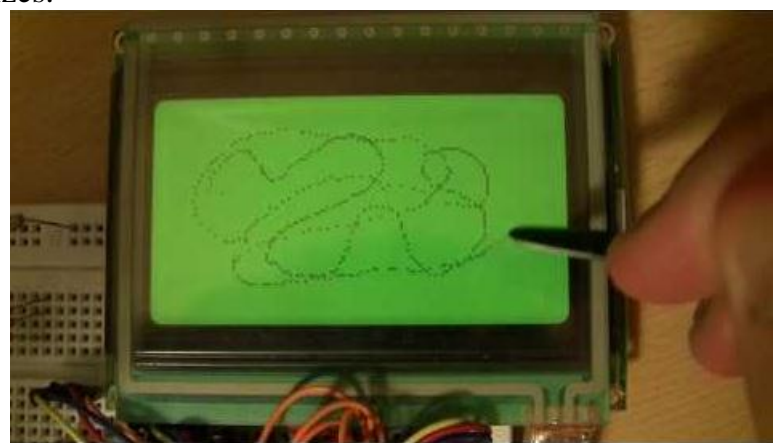

Fig. 5. Graphical LCD

This is a $128 \times 64$ liquid crystal display that support Chinese character, English characters and even graphics, very suitable for interactive work with ARM7. These LCDs have a graphics and a text mode, so we decided to use it in graphics mode and draw the text manually. This would only be practical on an ARM (or companionable) as we should need $1 \mathrm{~KB}(128 \times 64 / 8=1024$ bytes $)$ of memory for a screen buffer, plus memory for the characters.

The operation of the display was first implemented by turning it on by sending the instruction to the screen as shown in Table 1.

\begin{tabular}{|c|c|c|c|c|c|c|c|c|c|c|}
\hline Instruction & D/I & R/W & DB7 & DB6 & DB5 & DB4 & DB3 & DB2 & DB1 & DB0 \\
\hline Display & 0 & 0 & 0 & 0 & 1 & 1 & 1 & 1 & 1 & 1 \\
\hline
\end{tabular}

Table 1. Display Instruction

To draw any data on the screen, there are entire of 8192 pixels on a 128 X 64 pixel screen and every pixel is 
control by a sequence of instructions. The screen is split into its $\mathrm{X}$ and $\mathrm{Y}$ axis. The $\mathrm{Y}$ axis represents the line to which pixel should be written to and the $\mathrm{X}$ axis shows the column to which they will be written to. The followingstage is issuing a data write command, data bits which are high will be darkened on the $\mathrm{Y}$ address line in the $\mathrm{X}$ page. The driver scans over the pages, using the core $\mathrm{Y}$ address counter to its advantage and resetting the $\mathrm{X}$ page as it scans through the lines [1].

\section{E. ZIGBEE TRANSRECEIVER}

A WSN consists of many inexpensive wireless sensors, which are capable of collecting, storing, processing environmental information, and Communicating with neighbouring nodes. ZigBee/IEEE 802.15.4 is a global hardware and software Standard designed for WSN requiring high reliability, low cost, low power, scalability, And low data rate [4].

The Zigbee is the wireless sensor. It is use for the wireless communication between two nodes. The fig 6shows the zigbee wireless Trans-receiver sensor. In this present project the encoded drawn text pattern or images are transmitted towards the receiver side by using zigbee transmitter. The same type of zigbee wireless sensor is used at receiver side for receiving purpose.

ZigBee and IEEE 802.15.4 are designed for lightweight sensor platforms.ZigBee is designed to support low-cost network layer. In ZigBee, the network layer provides reliable and secure transmissions among devices [5].

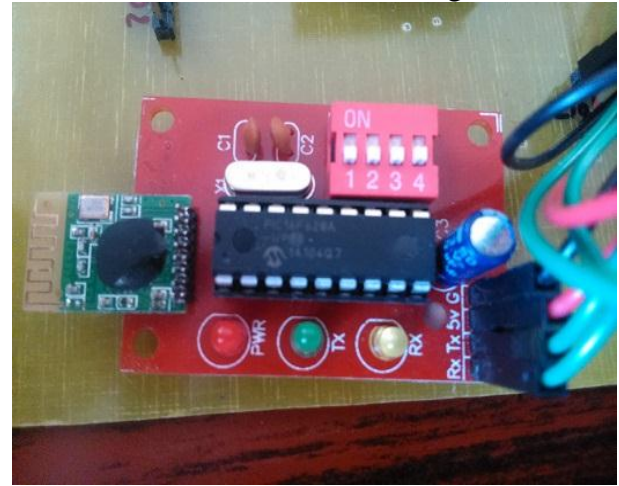

Fig. 6. Zigbee Trans-receiver Module

\section{SOFTWARE DESCRIPTION}

\section{A. Keil Micro-Vision Software:}

Keil Micro Vision-4 IDE is a robust editor and a windows based software development tool that integrates all tools like C compiler, macro assembler, linker/loader and Hex file creator. In this software we can write and edit the programs with the help of text editor and can convert the source code into HEX files with the help of Hex generator.

B. Viewing Code \& Data

- Mixed high level language and assembly code is shown in disassembly window. When the disassembly window is active window, then all debug stepping commands work on assembly level.

- System register values and project workplace is shown in register window. All registers and flags are updated and shown in register window after each instruction executes.

- Program symbols used in your applications are displayed on Symbol window hierarchy tab.

- The output of various debugger commands are shown in output window.

\section{Executing Code}

1. Reset: When we use reset button, $\mu$ Vision restarts the simulation using the new reset option.

2. Run/Stop: Use of Run/stop buttons and commands makes execution of code easy. Starting and stopping of the program execution may be controlled using commands you enter in the output window or using buttons on the toolbar.

3. Single stepping:

4. The "Step Over" button on the toolbar executes one line of C code ( single-step) at a time. The "Step into" button executes one line of $\mathrm{C}$ code (single-step) and it will step into function is a function call in encountered.

5. Breakpoint:

6. The "Insert/Remove breakpoint" button sets or unset an execution breakpoint on the current cursor line. The "Enable/Disable breakpoint" button enables or disables existing breakpoint on the current cursor line [1].

\section{RESULTS AND DISCUSSION \\ Algorithm}

1. Start.

2. Select the option a) draw the pattern b) select image.

3. If option (a) is select then draw the text or pattern on touchscreen display.

4. The X \& Y coordinates of the touch area detect and encoded by the arm7 processor.

5. The encoded $\mathrm{X} \& \mathrm{Y}$ coordinate send through zigbee module.

6. If option (b) is select then select the predefined images and send.

7. Repeat the step 3 to step 6 .

8. End.

B. Flow chart

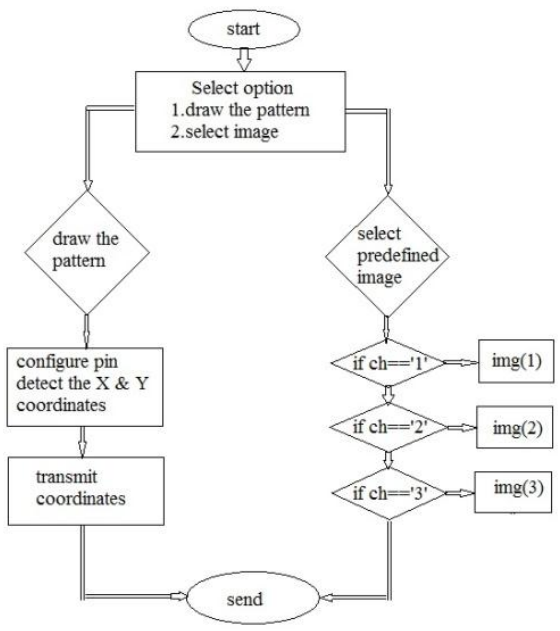

Fig 7. System flow chart 
C.

Project snapshot

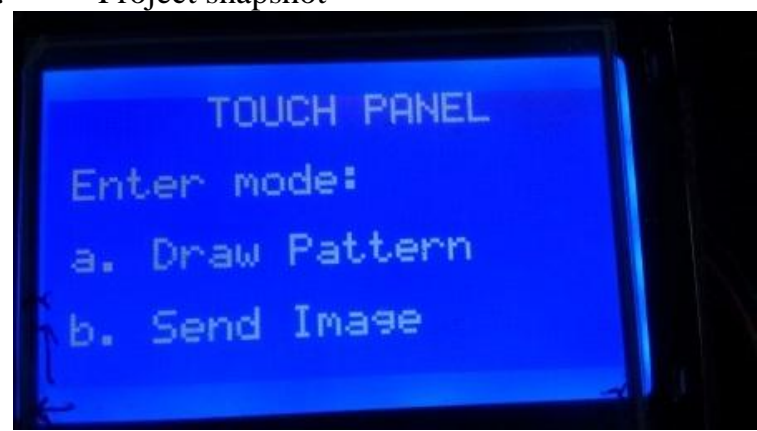

Fig 8.enter mode

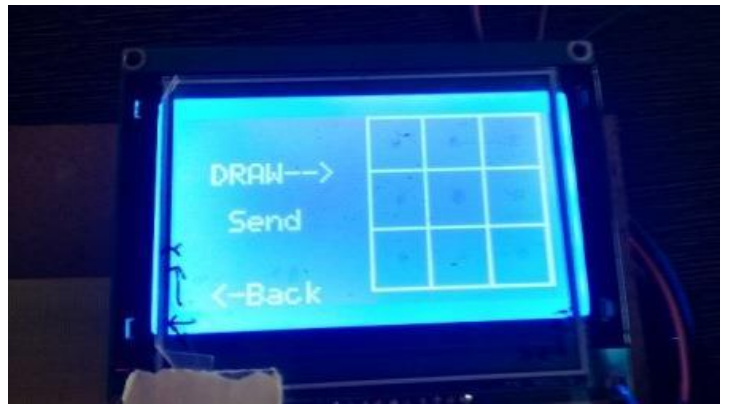

Fig9.draw pattern mode screen

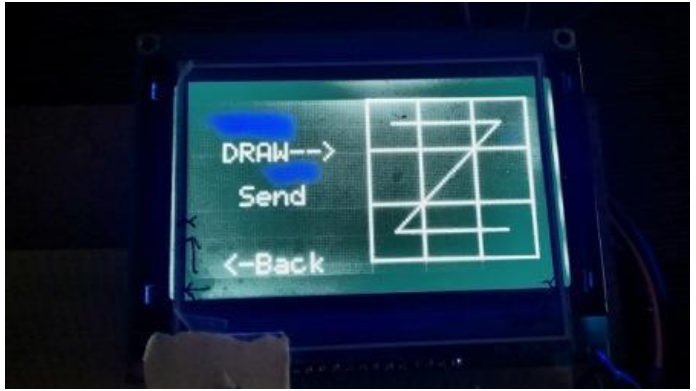

Fig 10.drawn pattern on screen (draw z pattern on screen)

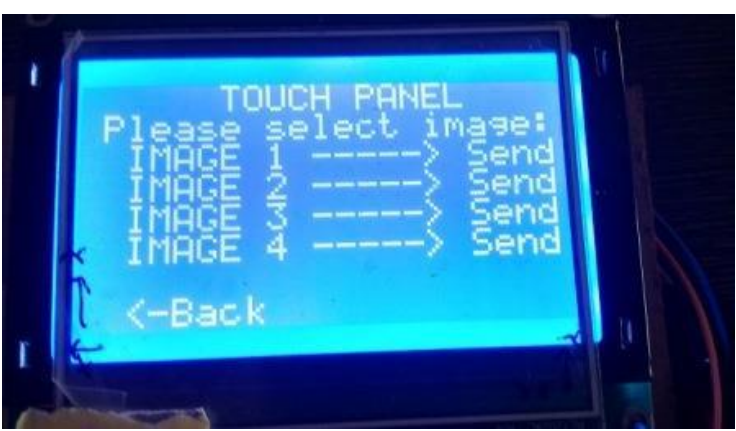

Fig 11.image selection mode screen

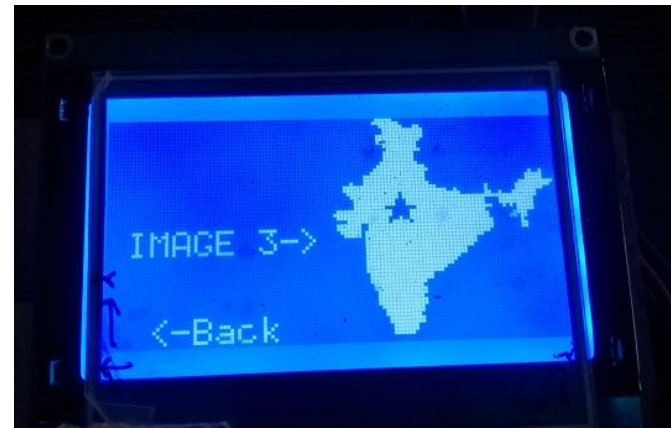

Fig 12.image selection mode (selection of image 3 )

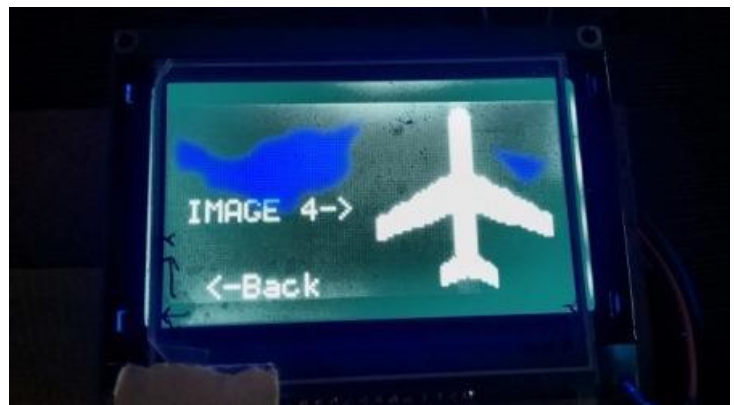

Fig 13.image selection mode (selection of image 4)

V.

\section{CONCLUSION}

We designed and implemented the ARM7 based wireless data transmission using zigbee in this paper. In this project the drawn pattern on touch screen display and the images which are stored in memory transmits using the zigbee wireless technology. And the transmitted pattern and images are display on graphic LCD at the receiver end. This project is useful for communication purpose where the voice data transmission is difficult like industries, chemical laboratories etc. The present work also used as enotice board in colleges, industries, departments etc.

\section{ACKNOWLEDGMENT}

We are sincerely thanking our project guide Prof. D.K. Shende for her guidance and valuable help throughout the development of our work.

\section{REFERENCES}

[1]. Shazeeda, Rajanna G. S., Pooja H. Matt, Anupama B. Gurappa, Monika D. Sharma, Nalini murlidhar.Touch screen based wireless data transmission through ARM processor. Sci.Int.(Lahore),25(4),713-717,2013

[2]. C. Sravani; N. V. N. Rao. Developing Touch Screen based Data Transmission Using S3C2440 Processor. Inter. J. Eng. Res. App... 2, 863-869 (2012).

[3]. Online available on www.circuitstoday.com

[4]. Online available on www.zigbee.org.

[5]. Wirelwss sensor network, An Information processing approch, Zhao Guibas

[6]. L. L. Delaney. Design of a Graphical LCD Driver and Educational LCD Primer. PhD diss., CornellUniversity, (2004). 